\title{
Early impact of depression symptoms on the decline in activities of daily living among older Japanese: Y-HALE cohort study
}

\author{
Mari Kazama $\cdot$ Naoki Kondo $\cdot$ Kohta Suzuki • \\ Junko Minai · Hisashi Imai · Zentaro Yamagata
}

Received: 21 July 2010/Accepted: 5 October 2010/Published online: 3 November 2010

(c) The Japanese Society for Hygiene 2010

\begin{abstract}
Objective It is well known that depression deteriorates basic activities of daily living (ADLs), such as eating and bathing, among the elderly, but little is known about the early impact of depression symptoms on the next higher level of functioning, namely higher-levels ADLs, such as instrumental self-maintenance, intellectual activities, and social roles. The objective of this study was to determine whether symptoms of depression are associated with a subsequent decline in higher-level ADLs within a 12-month period of time.

Methods The study cohort consisted of a random sample of 587 non-institutionalized adults aged $\geq 65$ years living in Yamanashi prefecture, Japan. The baseline survey was conducted in 2003. After 12 months, a mailed follow-up survey evaluated changes in higher-level ADLs (follow-up rate $98.6 \%$ ).
\end{abstract}

M. Kazama

Department of Nursing, Faculty of Nursing, Mejiro University,

Saitama, Saitama, Japan

M. Kazama · N. Kondo $(\square) \cdot$ K. Suzuki · J. Minai ·

Z. Yamagata

Department of Health Sciences,

Interdisciplinary Graduate School of Medicine and Engineering,

University of Yamanashi, 1110 Shimokato, Chuo,

Yamanashi 409-3898, Japan

e-mail: nkondo@yamanashi.ac.jp

J. Minai

School of Nursing, Odawara International University of Health and Welfare, Odawara, Kanagawa, Japan

H. Imai

Department of Business Administration,

Yamanashi Gakuin University, Kofu, Yamanashi, Japan
Results After adjusting for sociodemographic and behavioral confounders, logistic regression indicated that baseline severe depression symptoms were associated with a 3.2-fold (95\% confidence interval 1.6-6.3) higher chance of a subsequent decline in higher-level ADLs compared to those without severe depression symptoms. The presence of severe depression symptoms was selected by stepwise logistic regression in all models, except for the model with intellectual activities as an outcome, while other lifestyle factors were not selected.

Conclusions Symptoms of severe depression may adversely affect higher-level ADLs even in a relatively short time-frame. In addition, the early effects of depression symptoms may be stronger than those of other traditional lifestyle risk factors. Monitoring a wide range of ADLs in elderly individuals showing signs of depression may be important to prevent a functional decline in health and the need for long-term care.

Keywords Japan · Mental health · Aged - Depression . Disability

\section{Introduction}

Functional capacities of the elderly range from performing basic activities of daily living (ADLs) (e.g., eating, bathing, using the toilet, and dressing) to performing those higher than basic ADLs (higher-level ADLs), such as instrumental ADLs (e.g., money management and preparation of meals), intellectual activities (e.g., creative innovation, exploration, and recreation), and social roles (e.g., creative leadership and close/casual contact) [1]. To maintain their integration in society, the elderly require not only basic but also higher-level ADLs. 
The results of studies performed to date suggest that symptoms of depression predict declines in basic ADLs [2] and instrumental ADLs [3] as well as declines in intellectual activities and social role performances [4]. The decline may begin in the higher-level ADLs and proceed to deterioration of the more basic ADLs [5]. We previously reported that severe depression symptoms are related to subsequent declines in ADLs [4]. However, little is known about the early impact of depression symptoms on the decline in higher-level ADLs. For example, it is likely that a depressive mood will reduce the will of an individual to integrate into the society around him/herself. Therefore, we hypothesized that symptoms of depression may first affect higher-level ADLs, causing a deterioration in these activities within a relatively short period. In this study, we evaluated the early (i.e., within 12 months) impact of depression symptoms on higher-level ADLs among the elderly. In addition, given that mental illness may impede social integration and that lifestyle risk factors (such as cigarette smoking and physical inactivity) require an extended time (i.e., $>1$ year) to cause cardiovascular events (e.g., stroke and myocardial infarction that are other causes of functional disabilities), we also hypothesized that, within a short time-frame, the impact of depression on higher-level ADLs is stronger than those of traditional risk factors.

\section{Methods}

We used data from the Yamanashi Healthy Active Life Expectancy (Y-HALE) Study consisting of a random sample of 600 non-institutionalized residents of Yamanashi prefecture who were aged $\geq 65$ years. Those who were registered in governmental long-term care insurance programs were not eligible for enrollment in our study since we considered such individuals as already being disabled. The response rate was $98.3 \%$ ( $n=587,303$ men, 284 women). Trained investigators interviewed the subjects. Twelve months later, our postal survey evaluated the participants' higher-level ADLs and vital status. We had complete follow-up data for $579(98.6 \%)$ respondents. This study was approved by the Ethics Committee at the University of Yamanashi, School of Medicine. A detailed description of the study design and baseline characteristics of the subjects is provided elsewhere [6].

We evaluated high ADLs using the Tokyo Metropolitan Institute of Gerontology Index of Competence (TMIG-IC) [7]. This 13-item index has three components: instrumental self-maintenance, intellectual activity, and social role performance. Questions in the instrumental self-maintenance domain assess the abilities of instrumental ADLs, such as "Can you use a bus or a train by yourself?" and "Are you able to shop for daily necessities?". The intellectual activity domain includes questions such as "Are you able to fill out forms for your pension?" and "Do you read newspapers?", while questions in the social role component domain include those relevant to the maintenance of social life such as "Do you visit the homes of friends?" and "Are you sometimes called on for advice?". The participants are requested to answer either "1 (Yes)" or "0 (No)", and the summed scores indicate the higher-level ADLs. The validity and reliability of the TMIG-IC have been confirmed [7]. Following our previous approach [4], a decline in higher-level ADLs was defined as the reduction in TMIG-IC scores (of the total or of each section) at the follow-up. Our preliminary sensitivity analysis using other definitions of higher-level ADLs decline by different cutoff points did not largely alter the association between severe depression and a decline in higher-level ADLs, while we determined that our cutoff point showed the most sensitive results.

The mental health component $(\mathrm{MH})$ of the MOS 36Items Short Form Health Survey (SF-36) Japanese version 1.2 [8] was used to evaluate participants' baseline symptoms of depression. The score for the $\mathrm{MH}$ component can range from 0 to 100 based on the answers to five questions evaluating the feelings of nervousness, depression, and happiness, as well as peacefulness and calmness. Yamazaki et al. [9] reported that the $\mathrm{MH}$ score and the Zung Self-rating Depression Scale were strongly correlated (correlation coefficient -0.63) and that severe depression symptoms determined by the $\mathrm{MH}$ cutoff score of 52 had a sensitivity of $91.8 \%$ and a specificity of $84.6 \%$ relative to the Zung Scale.

\section{Statistical analysis}

The data on 530 subjects for whom the TMIG-IC scores at baseline and follow-up and the baseline $\mathrm{MH}$ score were available (=complete dataset) were used in our analysis. The baseline characteristics for those subjects for whom the information on the variables was complete and those for whom data were missing were not statistically different. We examined the differences in the incidence of a decline in higher-level ADLs within a 12-month period of time based on the presence of severe depression symptoms, sociodemographic factors (age, gender, family composition, income, and educational attainment), health status (hospitalization, chronic disease history determined by regular visits to a doctor, and physical health measured by the SF-36 Physical Component Summary score) [8], lifestyle factors (exercise habits, smoking, and alcohol intake), and baseline TMIG-IC score. We then used multiple logistic regression to model the association between baseline severe depressive symptoms and the incidence of a 
decline in higher-level ADLs, adjusting for multiple potential confounders. Severe depression symptoms were defined by the suggested SF-36 cutoff score of 52 [9]. Income and educational levels were modeled as categorical parameters using dummy variables, including the categories for missing data. This inclusion of samples with missing data did not significantly alter the point estimates of the main results, only their statistical significance. By using a stepwise logistic regression approach, we additionally evaluated if depression symptoms have a stronger impact on higher-level ADLs than other risk factors, such as lifestyle and socioeconomic status. We performed all analyses using SAS ver. 9.1 (SAS Institute, Cary, NC).

\section{Results}

The baseline analysis revealed that participants who had symptoms of severe depression were more likely to have a low income $(p=0.04)$ and to suffer from poor physical health $(p=0.0003)$ and chronic diseases $(p=0.10)$. At the 1-year follow-up, 89 subjects (17\%) had experienced a decline in higher-level ADLs. Among the subjects who showed signs of severe depression at baseline, 33\% had a decline in total TMIG-IC score at the 1-year follow-up; in comparison, only $15 \%$ of those without signs of severe depression experienced a decline in total TMIG-IC score $(p=0.001)($ Table 1)

Logistic regression demonstrated that the relative risk (RR) for a decline in higher-level ADLs in those showing signs of severe depression symptoms, compared to those without the symptoms, was 2.8 [95\% confidence interval (CI) 1.5-5.1] after adjusting for age, sex, and baseline TMIG-IC score. Further adjustments for family composition, socio-economic status, health status, lifestyle factors, and physical health did not largely change the estimate; the fully adjusted RR was 3.2 (95\% CI 1.6-6.3). When we evaluated the effect of severe depression symptoms on the three components of the TMIG-IC, the fully adjusted RRs (95\% CIs) were 6.8 (3.1-14.8), 1.8 (0.8-4.4), and 1.9 (0.9-4.2) for declines in social role function, intellectual activities, and instrumental selfmaintenance, respectively (Table 2). When we conducted stepwise logistic regression, in which age, sex, and baseline TMIG-IC score were forced to be included, severe depression symptoms were selected in all models except for the model using intellectual activities as outcome, while physical health was the single factor explaining the decline in intellectual activities (Table 2). In addition, sensitivity analyses using various specifications of covariates, including baseline TMIG-IC scores, confirmed that these findings were robust regardless of the specification of the covariates.

\section{Discussion}

Our results indicate that, among our participants, severe depression symptoms were strongly associated with an increased incidence of decline in higher-level ADLs within a 12-month period of time. The association was strongest with the highest level of ADLs (social role performance) and less strong with intellectual activities and instrumental self-maintenance. These associations were independent of an individual's socio-economic position, and other traditional risk factors did not largely alter the results. These findings support our hypothesis that severe depression symptoms begin to affect the ADLs of the elderly within 12 months - at the latest - of their appearance. In addition, the result of stepwise regression supported our hypothesis that within a relatively short time-frame, mental health may have a greater effect on the decline in higher-level ADLs than lifestyle factors.

The findings of this study support a potential psychological pathway linking depression and functional disabilities. As such, a persistent depressive mood, anxiety, impatience, and behavioral suppression may reduce an individual's desire to continue social activities, impeding access to necessary social support systems; it can also be a risk factor for disability and long-term care [10].

Based on the results of stepwise models, mental health may be less important to the maintenance of intellectual activities than physical health, or both mental and physical health need to perform jointly in order to maintain intellectual activities.

There are a number of potential limitations to this study. First, our self-reported measure of depression symptoms is not the same as that for a clinical diagnosis of depression. However, the results of a validation study suggest that the SF-36 MH component can be used in both epidemiologic research and clinical practice [9]. Second, our findings may be confounded by unobserved variables and temporal changes in explanatory variables. For example, although in our multivariate models we adjusted for baseline health status, health status could change over time. As such and despite our prospective observational approach, these results should be interpreted with caution in terms of their causality, since both depression symptoms and ADLs were time-varying variables and they could influence each other over time. Thus, further evaluations using repeated measurements are needed. Third, potential dropouts due to institutionalization before the follow-up survey was completed may have led to an underestimation of the estimated risk. In fact, the average TMIG-IC score slightly (0.63 points) increased after 12 months, which may be the consequence of vulnerable individuals dropping out of the study. Fourth, due to the insufficient incidence of a decline in higher-level ADLs, we were not able to conduct more 
Table 1 Characteristics of the study subjects at baseline and at the 1-year follow-up and the percentages of declines in higher-level activities of daily living during the 12-month study period by baseline characteristics

\begin{tabular}{|c|c|c|c|c|c|c|c|}
\hline \multirow[t]{3}{*}{ Variable (no. of missing datasets) } & \multicolumn{3}{|c|}{ Baseline characteristics } & \multicolumn{4}{|c|}{ Higher ADL decline at the follow-up, $n(\%)$} \\
\hline & \multirow{2}{*}{$\begin{array}{l}\text { Total } \\
n(\%)\end{array}$} & \multicolumn{2}{|c|}{ Depressive symptoms, $n(\%)$} & \multirow[b]{2}{*}{ Total score } & \multirow[b]{2}{*}{ Social role } & \multirow[b]{2}{*}{$\begin{array}{l}\text { Intellectual } \\
\text { activities }\end{array}$} & \multirow[b]{2}{*}{$\begin{array}{l}\text { Instrumental } \\
\text { self-maintenance }\end{array}$} \\
\hline & & Severe & Not severe & & & & \\
\hline \multicolumn{8}{|l|}{ Depressive symptoms (0) } \\
\hline Not severe & $475(90)$ & - & - & $71(15)$ & $42(9)$ & $37(8)$ & $57(12)$ \\
\hline Severe & $55(10)$ & - & - & $18(33)$ & $18(33)$ & $8(15)$ & $11(20)$ \\
\hline$p$ value & & & & 0.001 & $<0.0001$ & 0.09 & 0.09 \\
\hline \multicolumn{8}{|l|}{$\operatorname{Age}^{\mathrm{a}}(0)$} \\
\hline $65-74$ & $233(44)$ & $27(11)$ & $230(89)$ & $33(14)$ & $23(10)$ & $16(7)$ & $27(12)$ \\
\hline $75+$ & $297(56)$ & $37(11)$ & $293(89)$ & $56(19)$ & 37 (13) & $29(10)$ & $41(14)$ \\
\hline$p$ value & & 0.79 & & 0.15 & 0.35 & 0.23 & 0.45 \\
\hline \multicolumn{8}{|l|}{ Gender (0) } \\
\hline Male & $277(52)$ & $27(9)$ & $276(91)$ & 48 (17) & 39 (14) & $19(7)$ & $37(13)$ \\
\hline Female & $253(48)$ & 37 (13) & $247(87)$ & $41(16)$ & $21(8)$ & $26(10)$ & $31(12)$ \\
\hline$p$ Value & & 0.11 & & 0.72 & 0.03 & 0.16 & 0.70 \\
\hline \multicolumn{8}{|l|}{ Education (62) } \\
\hline$<$ High school & 199 (43) & $21(9)$ & $202(91)$ & 37 (19) & $22(11)$ & $17(9)$ & $28(14)$ \\
\hline High school+ & $269(57)$ & $34(12)$ & $260(88)$ & 43 (16) & $32(12)$ & $20(7)$ & $33(12)$ \\
\hline$p$ value & & 0.43 & & 0.45 & 0.78 & 0.66 & 0.57 \\
\hline \multicolumn{8}{|c|}{ Individual income; 1000 yen/month (68) } \\
\hline$<99$ & $199(43)$ & $29(13)$ & $188(87)$ & 37 (19) & $22(11)$ & $22(11)$ & $32(16)$ \\
\hline $100-199$ & $124(27)$ & $14(10)$ & $125(90)$ & $20(16)$ & $16(13)$ & $10(8)$ & $13(11)$ \\
\hline $200+$ & $139(31)$ & $8(5)$ & $141(95)$ & $19(14)$ & $14(11)$ & $5(4)$ & $12(9)$ \\
\hline$p$ value & & 0.04 & & 0.48 & 0.76 & 0.05 & 0.09 \\
\hline \multicolumn{8}{|l|}{ Family composition (1) } \\
\hline Living alone & $43(8)$ & $6(11)$ & $48(89)$ & $7(16)$ & $4(9)$ & $2(5)$ & $9(21)$ \\
\hline With spouse (2 people) & $255(43)$ & $24(10)$ & $220(90)$ & $32(14)$ & $25(11)$ & $16(7)$ & $24(11)$ \\
\hline $3+$ people & $261(49)$ & $34(12)$ & $253(88)$ & $50(19)$ & $31(12)$ & $26(10)$ & $35(13)$ \\
\hline$p$ value & & 0.76 & & 0.34 & 0.88 & 0.35 & 0.17 \\
\hline \multicolumn{8}{|l|}{ Physical health (PCS score ${ }^{\mathrm{b}}$ ) (2) } \\
\hline High & $267(51)$ & $18(6)$ & $275(94)$ & $38(14)$ & $24(9)$ & $36(12)$ & $29(11)$ \\
\hline Low & $261(49)$ & $45(15)$ & $246(85)$ & $51(20)$ & $35(13)$ & $55(19)$ & $39(15)$ \\
\hline$p$ value & & 0.0003 & & 0.10 & 0.11 & 0.03 & 0.16 \\
\hline \multicolumn{8}{|l|}{ Chronic disease (1) } \\
\hline Yes & $412(78)$ & $55(12)$ & $400(88)$ & $73(18)$ & $52(13)$ & $37(9)$ & $55(13)$ \\
\hline No & $117(22)$ & $9(7)$ & $120(93)$ & $16(14)$ & $8(7)$ & $8(7)$ & $13(11)$ \\
\hline$p$ value & & 0.10 & & 0.30 & 0.08 & 0.46 & 0.52 \\
\hline \multicolumn{8}{|l|}{ Smoking (0) } \\
\hline Never & $308(58)$ & $40(12)$ & $305(88)$ & $51(17)$ & $31(10)$ & $27(9)$ & $38(12)$ \\
\hline Ever & $158(30)$ & $18(11)$ & $150(89)$ & $29(18)$ & $22(14)$ & $14(9)$ & $22(13)$ \\
\hline Current & $64(12)$ & $6(8)$ & $67(92)$ & $9(14)$ & $7(11)$ & $4(6)$ & $8(13)$ \\
\hline$p$ value & & 0.70 & & 0.85 & 0.46 & 0.79 & 0.89 \\
\hline \multicolumn{8}{|l|}{ Alcohol drinking (0) } \\
\hline Never & $257(50)$ & 39 (13) & $256(87)$ & 45 (18) & $25(10)$ & $24(9)$ & $37(14)$ \\
\hline Less than $1 /$ week & $92(17)$ & $10(10)$ & $87(90)$ & $11(12)$ & $9(10)$ & $7(8)$ & $7(8)$ \\
\hline 1-6/week & $75(14)$ & $9(11)$ & $71(89)$ & $17(28)$ & $13(17)$ & $5(7)$ & $10(13)$ \\
\hline Every day & $106(20)$ & $6(5)$ & $109(95)$ & $16(15)$ & $13(12)$ & $9(9)$ & $14(13)$ \\
\hline
\end{tabular}


Table 1 continued

\begin{tabular}{|c|c|c|c|c|c|c|c|}
\hline \multirow[t]{3}{*}{ Variable (no. of missing datasets) } & \multicolumn{3}{|c|}{ Baseline characteristics } & \multirow{2}{*}{\multicolumn{4}{|c|}{ Higher ADL decline at the follow-up, $n(\%)$}} \\
\hline & \multirow{2}{*}{$\begin{array}{l}\text { Total } \\
n(\%)\end{array}$} & \multicolumn{2}{|c|}{ Depressive symptoms, $n(\%)$} & & & & \\
\hline & & Severe & Not severe & Total score & Social role & $\begin{array}{l}\text { Intellectual } \\
\text { activities }\end{array}$ & $\begin{array}{l}\text { Instrumental } \\
\text { self-maintenance }\end{array}$ \\
\hline$p$ value & & 0.14 & & 0.30 & 0.30 & 0.88 & 0.42 \\
\hline \multicolumn{8}{|l|}{ Exercise habits (1) } \\
\hline Yes & $298(56)$ & $34(11)$ & $288(89)$ & $48(16)$ & $36(12)$ & $25(9)$ & $35(11)$ \\
\hline No & $232(44)$ & $30(11)$ & $234(89)$ & $41(18)$ & $24(10)$ & $20(9)$ & $33(14)$ \\
\hline$p$ value & & 0.76 & & 0.63 & 0.53 & 0.92 & 0.39 \\
\hline
\end{tabular}

Higher ADLs at baseline and at the follow-up (mean \pm SD)

\begin{tabular}{|c|c|c|c|c|}
\hline & Baseline & & & At follow-up \\
\hline \multicolumn{5}{|l|}{ TMIG-IC ${ }^{\mathrm{c}}$ score (score range) } \\
\hline $\begin{array}{l}\text { Total score }(0-13) \\
p \text { value }\end{array}$ & $10.5 \pm 2.6$ & $\begin{array}{l}8.9 \pm 3.0 \\
<0.001\end{array}$ & $10.7 \pm 2.4$ & $11.2 \pm 2.5$ \\
\hline $\begin{array}{l}\text { Social role }(0-4) \\
p \text { value }\end{array}$ & $3.4 \pm 1.0$ & $\begin{array}{l}2.6 \pm 1.4 \\
<0.001\end{array}$ & $3.5 \pm 1.0$ & $3.5 \pm 0.9$ \\
\hline $\begin{array}{l}\text { Intellectual activities }(0-4) \\
\quad p \text { value }\end{array}$ & $3.5 \pm 0.9$ & $\begin{array}{l}3.0 \pm 1.1 \\
0.001\end{array}$ & $3.5 \pm 0.8$ & $3.6 \pm 0.8$ \\
\hline $\begin{array}{l}\text { Instrumental self-maintenance }(0-5) \\
\quad p \text { value }\end{array}$ & $3.7 \pm 1.3$ & $\begin{array}{l}3.3 \pm 1.6 \\
0.02\end{array}$ & $3.8 \pm 1.3$ & $3.0 \pm 1.3$ \\
\hline
\end{tabular}

$A D L$ Activities of daily living, $S D$ standard deviation

Chi-squared tests and $t$ tests were used to calculate $p$ values for frequency and mean values, respectively. Severe depressive symptoms were defined by the cutoff score (=52) of the SF-36, mental health component (mean 78.8, SD 19.7) following the suggestion by Yamazaki et al. [9]

${ }^{a}$ Mean age 76.4 years (SD 9.6 years)

b MOS 36-Items Short Form Health Survey (SF-36) Physical Component Summary score; high score indicates good health [8]

c Tokyo Metropolitan Institute of Gerontology Index of Competence

Table 2 Results of bivariate, multivariate, and stepwise logistic regressions: relative risks for a decline in higher-level ADLs by severe depression symptoms (bivariate and multivariate models) or by selected factors (stepwise model)

\begin{tabular}{|c|c|c|c|c|}
\hline Depression symptoms & Total score & Social role & $\begin{array}{l}\text { Intellectual } \\
\text { activities }\end{array}$ & $\begin{array}{l}\text { Instrumental } \\
\text { self-maintenance }\end{array}$ \\
\hline Severe depression symptoms (vs. not severe/none)—bivariate model & $2.8(1.5-5.1)$ & $5.0(2.6-9.6)$ & $2.0(0.9-4.6)$ & $1.8(0.9-3.8)$ \\
\hline Severe depression symptoms (vs. not severe/none)—multivariate model & $3.2(1.6-6.3)$ & $6.8(3.1-14.8)$ & $1.8(0.75-4.4)$ & $1.9(0.9-4.2)$ \\
\hline \multicolumn{5}{|l|}{ Explanatory variables selected by stepwise model } \\
\hline Severe depression symptoms (vs. not severe/none) & $3.4(1.74-6.5)$ & $7.6(3.6-16.1)$ & Not selected & $2.2(1.05-4.6)$ \\
\hline Physical health $\left(\right.$ PCS score $\left.{ }^{a}\right)=$ high (vs. low) & & & $0.5(0.26-0.97)$ & \\
\hline
\end{tabular}

Values are given as the relative risk with the $95 \%$ confidence intervals (CI) given in parenthesis

Age, sex, and baseline TMIG-IC component score were forced to be included when stepwise variable selections were conducted

${ }^{a}$ MOS SF-36 Physical Component Summary score, high score represents good health [8]

detailed evaluations, including the effect of milder depression symptoms and age- or gender-specific effects. Although our previous data categorized depression symptoms into four levels (i.e., none, mild, moderate, and severe) [4], we dichotomized the variable in the present study. The non-significant associations between age, gender and decline in ADLs may also be explained by the short follow-up period. Finally, baseline cognitive function, which was not formally controlled, may affect participants' responses to our interview at baseline.

In conclusion, our findings suggest that severe depression symptoms can result in a deterioration of higher-level ADLs in the elderly within 12 months or earlier and that this deterioration is known to predict a future reduction of 
more basic ADL. Given these findings, early interventions for elderly patients showing signs of depression are important, and a wide range of ADLs of such individuals should be monitored in order to prevent further functional disabilities and the need for long-term care.

Acknowledgments The baseline survey in this study was funded by the government of Yamanashi prefecture, Japan. This study was also supported in part by the Ministry of Education, Culture, Sports, Science and Technology, Japan. When this manuscript was written, N.K. was supported by the fellowship grant in the Abe Fellowship Program administered by the Social Science Research Council and the American Council of Learned Societies in cooperation with and with funds provided by the Japan Foundation Center for Global Partnership. These sponsors did not have any involvement in the study design, data collection, analysis, data interpretation, and the writing of this paper. We are grateful for the assistance provided by Sarah Hill.

Conflict of interest None declared.

\section{References}

1. Lawton MP, Brody EM. Assessment of older people: self-maintaining and instrumental activities of daily living. Gerontologist. 1969;9:179-86.
2. Penninx BW, Leveille S, Ferrucci L, van Eijk JT, Guralnik JM. Exploring the effect of depression on physical disability: longitudinal evidence from the established populations for epidemiologic studies of the elderly. Am J Public Health. 1999;89:1346-52.

3. Kivela SL, Pahkala K. Depressive disorder as a predictor of physical disability in old age. J Am Geriatr Soc. 2001;49:290-6.

4. Kondo N, Kazama M, Suzuki K, Yamagata Z. Impact of mental health on daily living activities of Japanese elderly. Prev Med. 2008;46:457-62.

5. Fujiwara Y, Shinkai S, Kumagai S, Amano H, Yoshida Y, Yoshida $\mathrm{H}$, et al. Longitudinal changes in higher-level functional capacity of an older population living in a Japanese urban community. Arch Gerontol Geriatr. 2003;36:141-53.

6. Kondo N, Minai J, Kazama M, Imai H, Yamagata Z. Engagement in a cohesive group and higher-level functional capacity of older adults: a case of a traditional Japanese rotating saving and credit association: Mujin. Soc Sci Med. 2007;64:2311-23.

7. Koyano W, Shibata H, Nakazato K, Haga H, Suyama Y. Measurement of competence: reliability and validity of the TMIG Index of competence. Arch Gerontol Geriatr. 1991;13:103-16.

8. Fukuhara S, Bito S, Green J, Hsiao A, Kurokawa K. Translation, adaptation, and validation of the SF-36 Health Survey for use in Japan. J Clin Epidemiol. 1998;51:1037-44.

9. Yamazaki S, Fukuhara S, Green J. Usefulness of five-item and three-item mental health inventories to screen for depressive symptoms in the general population of Japan. Health Qual Life Outcomes. 2005;3:48.

10. Penninx BW, Guralnik JM, Ferrucci L, Simonsick EM, Deeg DJ, Wallace RB. Depressive symptoms and physical decline in community-dwelling older persons. JAMA. 1998;279:1720-6. 